\title{
Bacteria associated with vascular wilt of poplar
}

\author{
Hanna Kwaśna ${ }^{1}$ · Wojciech Szewczyk ${ }^{1}$ - Marlena Baranowska ${ }^{2}$. Jolanta Behnke-Borowczyk ${ }^{1}$
}

Received: 5 January 2021 / Revised: 10 June 2021 / Accepted: 25 June 2021 / Published online: 2 July 2021

(c) The Author(s) 2021

\begin{abstract}
In 2017, a 560-ha area of hybrid poplar plantation in northern Poland showed symptoms of tree decline. Leaves appeared smaller, turned yellow-brown, and were shed prematurely. Twigs and smaller branches died. Bark was sunken and discolored, often loosened and split. Trunks decayed from the base. Phloem and xylem showed brown necrosis. Ten per cent of trees died in 1-2 months. None of these symptoms was typical for known poplar diseases. Bacteria in soil and in the necrotic base of poplar trunk were analyzed with Illumina sequencing. Soil and wood were colonized by at least 615 and 249 taxa. The majority of bacteria were common to soil and wood. The most common taxa in soil were: Acidobacteria (14.76\%), Actinobacteria (14.58\%), Proteobacteria (36.87) with Betaproteobacteria (6.52\%), (6.10\%), Comamonadaceae (2.79\%), and Verrucomicrobia (5.31\%). The most common taxa in wood were: Bacteroidetes (22.72\%) including Chryseobacterium (5.07\%), Flavobacteriales (10.87\%), Sphingobacteriales (9.40\%) with Pedobacter cryoconitis (7.31\%), Proteobacteria (73.79\%) with Enterobacteriales (33.25\%) including Serratia (15.30\%) and Sodalis (6.52\%), Pseudomonadales (9.83\%) including Pseudomonas (9.02\%), Rhizobiales (6.83\%), Sphingomonadales (5.65\%), and Xanthomonadales (11.19\%). Possible pathogens were Pseudomonas, Rhizobium and Xanthomonas. The potential initial, endophytic character of bacteria is discussed. Soil and possibly planting material might be the reservoir of pathogen inoculum.
\end{abstract}

Keywords Bacteria $\cdot$ Pathogens $\cdot$ Plantation $\cdot$ Poplar hybrids $\cdot$ Vascular wilt

\section{Introduction}

Poplars are distributed predominantly throughout the northern hemisphere. Because of their rapid growth, wild Populus spp. and their hybrids are currently planted over huge areas worldwide as ornamental plants for landscape greening, production of wood, and multiple industrial uses (Jansson and Douglas 2007).

Poplar's susceptibility to phytopathogens is the main obstacle to its exploitation (reviewed in Newcombe 1996). The most serious fungal pathogens include vascular and parenchymal colonizers (Kwaśna et al. 2021).

Communicated by Erko Stackebrandt.

Hanna Kwaśna

hanna.kwasna@up.poznan.pl

1 Department of Forest Pathology, Poznań University of Life Sciences, Wojska Polskiego 71c, 60-625 Poznań, Poland

2 Department of Silviculture, Poznań University of Life Sciences, Wojska Polskiego 71a, 60-625 Poznań, Poland
There are also a few bacteria pathogenic on poplar. Pseudomonas syringae Van Hall and Xanthomonas populi (Ridé) Ridé \& Ridé cause wilting, necrosis, rot, injury, tumours and cankers (Ridé 1993; Nesme et al. 1994; Yuki et al. 2013). Symptoms caused by $P$. syringae in combination with frost occur usually on the south side of trees, just above soil level, and the development of $P$. syringae in poplar bark is promoted by fluctuating temperatures (Ramstedt et al. 1994). Pseudomonas aeruginosa (Schröter) Migula appeared on poplar recently (Attila et al. 2008). It causes soft rot, which results in wilting, with death of trees occurring quickly, even in less than 48 h. Rhizobium radiobacter Beijerinck and van Delden [syn. Agrobacterium radiobacter (Beijerinck and van Delden) Conn, Agrobacterium tumefaciens (Smith and Townsend) Conn] causes crown gall disease by transferring and integrating bacterial DNA (T-DNA) into the plant genome. In the 1990s, the bacterial genus Brenneria was also reported to cause canker of trees including poplar (Biosca et al. 2006). Currently, large portions of the plantation areas of the hybrid poplar Populus $\times$ euramericana in China and Hungary are affected by potentially lethal Lonsdalea populi (formerly Lonsdalea quercina subsp. populi) 
(Toth et al. 2013; Li et al. 2014; Li and He 2019). Bacteria overwinter on infected plant tissues, in necrosis, gummosis and sap oozing from wounds or healthy looking plant tissues.

Bacteria spread by windblown water droplets, contaminated tools, insects and animals. Infection is through roots, leaf scars and fresh wounds on branches and stems. The extent of symptoms depends on tree susceptibility. Planting highly resistant clones from selections and breeding programs is the only way to control bacterial disease.

In 2017, 560 ha of plantation of hybrid poplar (P. deltoides $\times$ P. nigra) in northern Poland showed symptoms of tree decline. Leaves of diseased trees appeared smaller, turned yellow-brown, and were shed prematurely. Twigs and smaller branches died without definite cankers. Bark of the entire trunk was sunken and discolored, often loosened and split. It often fell off, exposing wet wood. Trunks decayed from the base. The phloem showed brown necrosis. Ten per cent of trees died in 1-2 months (in June) after the first appearance of symptoms. None of the observed symptoms was typical for known poplar diseases. The possible contribution of vascular and parenchymal fungal pathogens has been suggested (Kwaśna et al. 2021).

The objectives of this research were to study: (1) the abundance and diversity of bacteria in soil and wood that may possibly contribute to development of vascular wilt in poplar, and (2) interactions among these bacteria and environmental conditions.

\section{Materials and methods}

\section{Site and sampling}

The study was carried out in Łoża, Czarne District, Człuchów County, Pomeranian Voivodeship, northern Poland $\left(53^{\circ} 41^{\prime} 29^{\prime \prime} \mathrm{N} 17^{\circ} 04^{\prime} 19^{\prime \prime} \mathrm{E}\right)$, in 560 ha of plantations of 5-6-year-old hybrid poplar ( $P$. deltoides $\times P$. nigra, cultivar AF2, from Italy) showing symptoms of crown decline and trunk-base decay (520 ha) and tree death (40 ha).

Trees were grown at a density of 425 trees/ha $(4 \times 4 \mathrm{~m}$ spacing), and had mean diameter of $9-10 \mathrm{~cm}$ at breast height. The post-agricultural soil was sandy loam, consisting of sand $(60 \%)$, silt $(20 \%)$ and clay $(20 \%)$, with low humus level, and with $\mathrm{pH}$ 6.5. The former crop was rye (Secale cereale $\mathrm{L}$.). The average temperature is $7.9^{\circ} \mathrm{C}$ and rainfall $680 \mathrm{~mm}$.

The understorey vegetation included Achillea millefolium L., Agrostis stolonifera L., Artemisia absinthium L., Artemisia vulgaris L., Cichorium intybus L., Elymus repens (L.) Gould, Lamium purpureum L., Lolium perenne L., Papaver rhoeas L., Poa annua L., Poa pratensis L., Poa trivialis L., Polygonum aviculare L., Polypodium vulgare
L., Polytrichum commune Hedw., Stellaria media Hist. Pl. Dauphiné, Taraxacum officinale F.H. Wigg., and Trifolium arvense $\mathrm{L}$.

Five wood cores, c. $10 \mathrm{~cm}$ long and $3 \mathrm{~cm}$ diameter, each including bark, phloem and xylem, were sampled from the bases of necrotic trunks of five symptomatic trees, $0 \mathrm{~cm}$ and $50 \mathrm{~cm}$ above the soil surface, with a Pressler borer. The core samples were surface-sterilized before being ground to sawdust with a cordless SPARKY BUR2 15E drill. In addition, five subsamples of soil were taken as cylindrical cores, $10 \mathrm{~cm}$ long and $5 \mathrm{~cm}$ diameter, from the surroundings of roots of five symptomatic trees. They were placed in sterile glass containers and refrigerated for $48 \mathrm{~h}$. There were no healthy, asymptomatic, trees in plantation which could be analyzed as the control.

\section{DNA extraction, amplification and Illumina sequencing}

Five samples of sawdust were prepared from five wood cores in a SPEX ${ }^{\mathrm{TM}}$ SamplePrep ${ }^{\mathrm{TM}}$ Freezer/Mill ${ }^{\mathrm{TM}}$ cryogenic mill. Genomic DNA from wood was extracted in $100 \mathrm{mg}$ of sawdust from each of the five wood cores with GeneJET ${ }^{\text {TM }}$ Plant Genomic DNA Purification Mini Kit (Thermo Scientific, USA). Genomic DNA from soil was extracted in $300 \mathrm{mg}$ of soil from each of the five soil cores with DNeasy PowerSoil Pro Kit (Qiagen, Hilden, Germany). The 16S rDNA from each subsample was amplified with bacteria-specific primers: 16S-F 5' CAGCCTACGGGNGGCWGCAG and 16S-R 5' ACAGGACTACHVGGGTATCTAATCC. Each amplification reaction was carried out in a final volume of $25.0 \mu \mathrm{L}$ containing $2 \mu \mathrm{L}$ DNA, $0.2 \mu \mathrm{L}$ of each primer, $10.1 \mu \mathrm{L}$ deionized water and $12.5 \mu \mathrm{L} 2 \mathrm{X}$ PCR MIX (A \& A Biotechnology, Gdynia, Poland). DNA amplification was performed under the following conditions: denaturation at $94{ }^{\circ} \mathrm{C}$ for $5 \mathrm{~min}$, followed by 35 cycles of denaturation at $94{ }^{\circ} \mathrm{C}$ for $30 \mathrm{~s}$, annealing at $56^{\circ} \mathrm{C}$ for $30 \mathrm{~s}$, elongation at $72^{\circ} \mathrm{C}$ for $30 \mathrm{~s}$, and a final elongation at $72{ }^{\circ} \mathrm{C}$ for $7 \mathrm{~min}$. Visualization of 5- $\mu \mathrm{l}$ amplicons was performed in $1.0 \%$ agarose gel dyed with Midori Green Advance DNA (Genetics). Pooled PCR products were purified using a MinElute PCR Purification Kit (Qiagen, Hilden, Germany). The concentration of PCR products was quantified using a Qubit 2.0 Fluorometer (Life Technologies, Carlsbad, CA, USA), and an equimolar mix of PCR products from each sample was prepared. The PCR product from each subsample was purified and sequenced with the use of Illumina SBS technology (Genomed S.A. Warsaw, Poland).

\section{Bioinformatics analysis}

A table of Operational Taxonomic Units (OTUs) was prepared by PIPITS, version 1.2.0 (Gweon et al. 2015). 
Read-pairs were joined with PEAR, version 0.9.6 (Zhang et al. 2014), filtered with a quality threshold of $q=30$ by FASTX-toolkit, version 0.0.13 (http://hannonlab.cshl.edu/ fastx_toolkit/index.html), converted to Fasta format and merged into a single file. Prepared sequences were de-replicated and subregions of ITS were selected with the use of ITSx, version 1.0.11 (Bengtsson-Palme et al. 2013). Unique sequences and those shorter than $100 \mathrm{bp}$ were removed. Remaining sequences were clustered with $97 \%$ sequence identity. The resulting representative sequences for each cluster were subjected to chimera detection and removal using the UNITE UCHIME reference data set, version 6.0 (https://unite.ut.ee/index.php). The input sequences were then mapped onto the representative sequences and taxonomy assigned using RDP Classifier, version 2.10.2 (Wang et al. 2007), against UNITE fungal ITS reference database, version 11.2 (Cole et al. 2014). This process resulted in the creation of a table of OTUs. Sequences were identified by comparison with reference sequences from the National Center for Biotechnology Information (NCBI) database.

Abundance of bacteria was defined as the average number of OTUs from five subsamples. Frequency of an individual taxon was defined as percentage (\%) of OTUs in the total number of OTUs from five subsamples. Diversity of bacterial community (\%) evaluated with the number of taxa in soil or wood is shown by a heatmap.

\section{Statistical analyses}

Differences in abundance of bacteria in soil and wood were analyzed with Chi-squared tests $\left(\chi^{2}\right)$. Diversity between communities of microfungi was compared with Margalef's diversity index $\left(D_{\mathrm{Mg}}\right)$, Shannon's diversity index $\left(H^{\prime}\right)$, Simpson's diversity index $(D)$, Shannon's evenness index $(E)$ and Berger-Parker's index (d) (Magurran 1988).

\section{Results}

A total of 34,866 and 21,207 OTUs were obtained, respectively, from the soil and wood of Populus hybrid using the Illumina sequencing technique (Table 1). The majority of bacteria were classified to the higher taxa. Classification resulted from the length of the sequence. The frequency of classified and non-classified bacteria was $96.57 \%$ and $3.43 \%$ in soil and $99.84 \%$ and $0.16 \%$ in wood. The frequency of bacteria absent from NCBI database was $0.70 \%$ and $0.63 \%$ in soil and wood, respectively. Soil and wood were colonized by at least 615 and 249 taxa. There were 242 non-identified taxa in soil and 16 in wood. The majority of bacteria were common to soil and wood.

The most common taxa in soil were: Acidobacteria (with Acidobacteriales and Solibacterales), Actinobacteria (with
Acidimicrobiales), Bacteroidetes (with Cytophagales, Flavobacteriales, Saprospirales and Sphingobacteriales), Chloroflexi (with Caldilineales), Nitrospirae, Planctomycetes, Proteobacteria (with Betaproteobacteria, Burkholderiales, Pseudomonadales, Rhizobiales, Sphingomonadales and Syntrophobacterales) and Verrucomicrobia (with Chthoniobacterales and Pedosphaerales).

The most common taxa in wood were: Bacteroidetes (with Flavobacteriales and Sphingobacteriales), Proteobacteria (with Burkholderiales, Enterobacteriales, Pseudomonadales, Rhizobiales, Sphingomonadales and Xanthomonadales).

Most taxa were present in wood of the diseased poplars and in soil. Potential pathogens were species in genera Pseudomonas and Xanthomonas. Dubious and unexpected species include Pedobacter cryoconitis and Sodalis.

Margalef's index $\left(D_{\mathrm{Mg}}\right)$ and Shannon's diversity index $\left(H^{\prime}\right)$ indicate greater diversity in soil than in wood, although Simpson's diversity index $(D)$ suggests more diversity in wood (Table 1). Conversely, Shannon's evenness index $(E)$ shows more evenness in communities in wood, although Berger-Parker's dominance index $(d)$ shows more dominance of individual taxa in wood.

\section{Discussion}

Research on bacteria has usually been focused on the study of single species. However, bacteria are social organisms and they occur in communities. Recent advances in genomics and molecular techniques have led to discovery and characterization of a vast bacterial diversity and to understanding of mutual interactions among them.

Illumina sequencing of $16 \mathrm{~S}$ rRNA genes was applied for studies of the bacteria associated with vascular wilt of poplar. Sequencing of the 16S rRNA gene is highly useful for bacterial classification because of its presence in almost all bacteria, its ability to exist as a multigene family or operon, its resistance to change over time, and its size (1500 bp long).

However, the 16S rRNA genes are highly conserved and do not provide sufficient resolution at lower taxon levels (i.e., species or strain). Thus, most bacteria in our studies were classified to the higher taxonomic ranks, making them ecologically and functionally indistinguishable. More precise taxonomic resolution was not possible because of: (1) insufficient number of sequences of the lower taxa in nucleotide databases, (2) occurrence of species sharing similar and/ or identical 16S rRNA sequences, (3) nomenclature problems arising from multiple genomovars assigned to single species or complexes, and (4) the possible occurrence of new taxa. These reasons undoubtedly affected the scientific completeness of the research presented. Therefore, in many cases, the data are discussed and interpreted with caution 
Table 1 Bacteria present in soil and wood of wilted poplar

\begin{tabular}{|c|c|c|c|c|}
\hline \multirow{2}{*}{ No. } & \multirow{2}{*}{ Taxon } & \multirow{2}{*}{ Order } & \multicolumn{2}{|c|}{ Frequency (\%) } \\
\hline & & & Soil 1 & Wood \\
\hline \multicolumn{5}{|c|}{ Phylum Acidobacteria } \\
\hline 1. & $\begin{array}{l}\text { Acidobacteria }(5.281 \%, 0.124 \%)^{\mathrm{a}} \text {, Acidobacteriaceae, Candidatus Koribacter, Chloracidobacteria }(5.764 \% \text {, } \\
0.055 \%) \text {, Koribacteraceae }(1.237 \%, 0.002 \%) \text {, Terriglobus }\end{array}$ & Acidobacteriales & 12.385 & 0.286 \\
\hline 2. & Nitrosopumilus & Cenarchaeales & 0.005 & \\
\hline 3. & Geothrix $(0.021 \%, 0 \%)$, Holophagaceae & Holophagales & 0.042 & \\
\hline \multirow[t]{3}{*}{4.} & Bryobacteraceae, Candidatus Solibacter $(1.019 \%, 0.005 \%)$, Solibacteres $(1.097 \%, 0.022 \%)$ & Solibacterales & 2.325 & 0.030 \\
\hline & Frequency & & 14.757 & 0.316 \\
\hline & Number of taxa (13) & & 12 & 7 \\
\hline \multicolumn{5}{|c|}{ Phylum Actinobacteria } \\
\hline 1. & $\begin{array}{l}\text { Actinobacteria (8.880\%, 0.059\%), Actinocorallia, Actinoplanes, Aeromicrobium, Agromyces, Arthrobacter, } \\
\text { Catellatospora, Cellulomonas, Cellulomonas xylanilytica, Corynebacterium, Dactylosporangium, Frankiaceae, } \\
\text { Geodermatophilaceae, Iamia, Intrasporangiaceae, Kineosporiaceae, Kribbella, Lentzea, Microbacteriaceae, } \\
\text { Micrococcaceae, Microlunatus, Micromonosporaceae, Mycobacterium, Nakamurellaceae, Nocardioidaceae, } \\
\text { Nocardioides, Nonomuraea, Phycicoccus, Pilimelia, Pimelobacter, Propionibacteriaceae, Pseudonocardia, } \\
\text { Rhodococcus, Salinibacterium, Sporichthya, Sporichthyaceae, Streptacidiphilus, Streptomyces, Terracoccus }\end{array}$ & Acidimicrobiales & 13.896 & 1.399 \\
\hline 2. & Gaiellaceae, Gaiellales & Gaiellales & 0.513 & 0.007 \\
\hline \multirow[t]{3}{*}{3.} & Conexibacteraceae, Patulibacteraceae, Solirubrobacteraceae, Solirubrobacterales & Solirubrobacterales & 0.174 & 0.003 \\
\hline & Frequency & & 14.583 & 1.409 \\
\hline & Number of taxa (46) & & 46 & 28 \\
\hline \multicolumn{5}{|c|}{ Phylum Armatimonadetes } \\
\hline 1. & Chthonomonadaceae, Chthonomonadete & Chthonomonadales & 0.091 & \\
\hline \multirow[t]{3}{*}{2.} & Fimbriimonadaceae, Fimbriimonadales, Fimbriimonas & Fimbriimonadales & 0.285 & 0.020 \\
\hline & Frequency & & 0.376 & 0.020 \\
\hline & Number of taxa (5) & & 5 & \\
\hline \multicolumn{5}{|c|}{ Phylum Bacteroidetes } \\
\hline 1. & $\begin{array}{l}\text { Adhaeribacter, Cytophaga, Cytophagaceae }(1.526 \%, 0.026 \%), \text { Dyadobacter, Flectobacillus, Larkinella, } \\
\text { Spirosoma, Sporocytophaga }\end{array}$ & Cytophagales & 1.705 & 1.949 \\
\hline \multirow[t]{2}{*}{2.} & $\begin{array}{l}\text { Chryseobacterium (0.014\%, 5.074\%), Crocinitomix, Cryomorphaceae, Flavobacteriaceae, Flavobacterium } \\
(3.055 \%, 5.477 \%) \text {, Flavobacterium columnare, Flavobacterium frigidarium, Flavobacterium succinicans, }\end{array}$ & Flavobacteriales & 3.482 & 10.873 \\
\hline & Fluviicola & & & \\
\hline 3. & Rubricoccus & Rhodothermales & 0.016 & \\
\hline 4. & $\begin{array}{l}\text { Haliscomenobacter, Flavihumibacter, Flavisolibacter, Chitinophagaceae }(2.583 \%, 0.252 \%) \text {, Niabella, } \\
\text { Saprospira, Saprospiraceae, Sediminibacterium }\end{array}$ & Saprospirales & 3.301 & 0.504 \\
\hline \multirow[t]{3}{*}{5.} & $\begin{array}{l}\text { Pedobacter }(0.039 \%, 1.725 \%), \text { Pedobacter cryoconitis }(0.004 \%, 7.306 \%) \text {, Sphingobacteriaceae, } \\
\text { Sphingobacterium faecium }\end{array}$ & Sphingobacteriales & 1.709 & 9.396 \\
\hline & Frequency & & 10.213 & 22.722 \\
\hline & Number of taxa (33) & & 30 & 26 \\
\hline \multirow{4}{*}{1.} & Phylum Chlamydiae & & & \\
\hline & Candidatus Rhabdochlamydia, Chlamydiales & Chlamydiales & 0.016 & \\
\hline & Frequency & & 0.016 & \\
\hline & Number of taxa (2) & & 2 & 1 \\
\hline & \multicolumn{4}{|l|}{ Phylum Chlorobi } \\
\hline \multirow[t]{3}{*}{1.} & Chlorobi & & 0.041 & 0.001 \\
\hline & Frequency & & 0.411 & 0.001 \\
\hline & Number of taxa (1) & & 1 & 1 \\
\hline \multicolumn{5}{|c|}{ Phylum Chloroflexi } \\
\hline 1. & Ardenscatena & Ardenscatenales & 0.009 & \\
\hline 2. & Anaerolinaceae, Anaerolineae $(1.209 \%, 0.004 \%)$ & Anaerolineales & 1.221 & 0.004 \\
\hline 3. & Caldilinea, Caldilineaceae, Chloroflexi $(2.575 \%, 0.002 \%)$ & Caldilineales & 2.585 & 0.020 \\
\hline 4. & Herpetosiphonales & Herpetosiphonales & 0.013 & \\
\hline 5. & Ktedonobacteraceae & Ktedonobacterales & 0.010 & \\
\hline \multirow[t]{3}{*}{6.} & Kouleothrixaceae, Roseiflexales, Thermomicrobia & Roseiflexales & 0.203 & 0.004 \\
\hline & Frequency & & 4.041 & 0.028 \\
\hline & Number of taxa (11) & & 11 & 4 \\
\hline & \multicolumn{4}{|l|}{ Phylum Cyanobacteria } \\
\hline \multirow[t]{3}{*}{1.} & Chlorophyta, Cyanobacteria, Stramenopiles, Streptophyta & & 0.066 & 0.016 \\
\hline & Frequency & & 0.066 & 0.016 \\
\hline & Number of taxa (4) & & 4 & 1 \\
\hline & Phylum Elusimicrobia & & & \\
\hline 1. & Elusimicrobia, Elusimicrobiales & Elusimicrobiales & 0.163 & \\
\hline
\end{tabular}

and conclusions formulated with care. Only assumptions can be made.

In wood of diseased poplar the most common taxa of bacteria were: Bacteroidetes, particularly Chryseobacterium and Flavobacterium; Proteobacteria including Enterobacteriales, particularly Serratia and Sodalis; Pseudomonadales; Rhizobiales; Sphingobacteriales including Pedobacter cryoconitis; Xanthomonadales.Each of these taxa includes species or strains with various trophisms and functions: endophytes, pathogens or saprotrophs. It is not easy to draw a clear distinction between pathogens and non-pathogens. They occupy the same ecological niches and possess similar mechanisms for plant colonization. Endophytic, pathogenic and saprotrophic strains are often found within the same species, and the incidence and severity of potential diseases are affected by additional factors, including host vigour, environmental conditions and host-pathogen specific interactions (Schulz et al. 1999). 
Table 1 (continued)

\begin{tabular}{|c|c|c|c|c|}
\hline & Frequency & & 0.163 & \\
\hline & Number of taxa (2) & & 2 & \\
\hline \multicolumn{5}{|c|}{ Phylum Fibrobacteres } \\
\hline \multirow[t]{3}{*}{1.} & Fibrobacteria & & 0.020 & 0.001 \\
\hline & Frequency & & 0.020 & 0.001 \\
\hline & Number of taxa (1) & & 1 & 1 \\
\hline \multicolumn{5}{|c|}{$\begin{array}{cc} & \text { Phylum Firmicutes } \\
\end{array}$} \\
\hline \multirow[t]{3}{*}{1.} & $\begin{array}{l}\text { Bacillales, Bacillus, Bacillus flexus, Bacillus muralis, Clostridium, Paenibacillus, Paenibacillus chondroitinus, } \\
\text { Sporosarcina }\end{array}$ & Bacillales & 0.207 & 0.017 \\
\hline & Frequency & & 0.207 & 0.017 \\
\hline & Number of taxa (8) & & 8 & \\
\hline \multicolumn{5}{|c|}{$\begin{aligned} & \text { Phylum Gemmatimonadetes } \\
&\end{aligned}$} \\
\hline \multirow[t]{3}{*}{1.} & Gemmatimonadales, Gemmatimonadetes $(1.137 \%, 0 \%)$, Gemmatimonas & Gemmatimonadales & 1.574 & 0.004 \\
\hline & Frequency & & 1.574 & 0.004 \\
\hline & Number of taxa (3) & & 3 & \\
\hline & $\begin{array}{c}\text { Phylum Nitrospirae } \\
\end{array}$ & & & \\
\hline \multirow[t]{3}{*}{1.} & Leptospirillaceae, Nitrospira $(1.912 \%, 0.011 \%)$, Nitrospiraceae, Thermodesulfovibrio naceae & Nitrospirales & 3.452 & 0.015 \\
\hline & Frequency & & 3.452 & 0.015 \\
\hline & Number of taxa (5) & & 5 & 2 \\
\hline \multicolumn{5}{|c|}{ Phylum Planctomycetes } \\
\hline 1. & Gemmataceae, Isosphaeraceae & Gemmatales & 0.438 & 0.009 \\
\hline 2. & Phycisphaerae & Phycisphaerales & 1.583 & 0.017 \\
\hline 3. & Pirellula, Pirellulaceae & Pirellulales & 0.666 & 0.009 \\
\hline \multirow[t]{3}{*}{4.} & Planctomyces, Planctomycetes & Planctomycetales & 1.113 & 0.008 \\
\hline & Frequency & & 3.800 & 0.043 \\
\hline & Number of taxa (7) & & 7 & \\
\hline \multicolumn{5}{|c|}{$\begin{array}{ll} & \text { Phylum Proteobacteria } \\
\end{array}$} \\
\hline 1. & Alpha-, Beta- $(6.516 \%, 0.105 \%)$, Delta-, Gammaproteobacteria & & 7.554 & 0.139 \\
\hline 2. & Alteromonadales, Cellvibrio & Alteromonadales & 0.061 & 0.052 \\
\hline 3. & Bacteriovoracaceae, Bdellovibrio bacteriovorus, Bdellovibrio & Bdellovibrionales & 0.265 & 0.03 \\
\hline \multirow[t]{2}{*}{4.} & Acidovorax, Alcaligenaceae, Burkholderia, Burkholderia andropogonis (0\%, 1.057\%), Comamonadaceae & Burkholderiales & 6.102 & 3.528 \\
\hline & $\begin{array}{l}(2.786 \%, 0.884 \%), \text { Herminiimonas, Hydrogenophaga, Janthinobacterium, Janthinobacterium lividum, } \\
\text { Methylibium, Oxalobacteraceae }(1.709 \%, 0.379 \%) \text {, Paucibacter, Polaromonas, Ramlibacter, Rubrivivax, } \\
\text { Variovorax, Variovorax paradoxus }\end{array}$ & & & \\
\hline 5. & Asticcacaulis biprosthecium, Caulobacter, Caulobacteraceae, Mycoplana, Phenylobacterium & Caulobacterales & 0.549 & 1.417 \\
\hline 6. & Chromatiales & Chromatiales & 0.007 & \\
\hline 7. & Desulfuromonadales, Geobacter, Pelobacteraceae & Desulfuromonadales & 0.377 & \\
\hline 8. & $\begin{array}{l}\text { Enterobacteriaceae }(0.012 \%, 11.349 \%) \text {, Serratia }(0.005 \%, 15.303 \%) \text {, Serratia marcescens, Sodalis }(0.001 \% \text {, } \\
6.524 \%) \text {, Entotheonellaceae, Erwinia, Rahnella aquatilis }\end{array}$ & Enterobacteriales & 0.018 & 33.247 \\
\hline 9. & Aquicella, Coxiellaceae, Legionellales & Legionellales & 0.028 & \\
\hline 10. & Crenothrix & Methylococcales & 0.011 & \\
\hline 11. & Methylophilaceae & Methylophilales & 0.598 & 0.087 \\
\hline 12. & Chondromyces, Haliangiaceae, Myxococcales (1.604\%, 0.102\%), Plesiocystis, Polyangiaceae & Myxococcales & 2.129 & 0.111 \\
\hline 13. & Nitrosomonadales, Nitrosovibriotenuis & Nitrosomonadales & 0.158 & 0.001 \\
\hline 14. & Halomonadaceae & Oceanospirillales & 0.010 & \\
\hline 15. & Acinetobacter, Moraxellaceae, Perlucidibaca, Pseudomonas (3.065\%, 9.017\%), Pseudomonas viridiflava & Pseudomonadales & 3.150 & 9.829 \\
\hline 16. & $\begin{array}{l}\text { Afifella, Aminobacter, Aurantimonadaceae, Balneimonas, Beijerinckiaceae, Boseageno, Bradyrhizobiaceae, } \\
\text { Devosia, Hyphomicrobiaceae, Hyphomicrobium, Kaistia, Labrys, Mesorhizobium, Methylobacterium } \\
\text { adhaesivum, Parvibaculum, Pedomicrobium, Phyllobacteriaceae, Phyllobacterium, Pleomorphomonas, } \\
\text { Rhizobiaceae (0.095\%, 5.724\%), Rhizobium, Rhizobium leguminosarum, Rhodoplanes (1.807\%, 0.034\%), } \\
\text { Xanthobacteraceae }\end{array}$ & Rhizobiales & 4.792 & 6.826 \\
\hline 17. & Hyphomonadaceae, Rhodobacter, Rhodobacteraceae & Rhodobacterales & 0.581 & 0.030 \\
\hline 18. & Azoarcus, Dechloromonas, Rhodocyclaceae, Uliginosibacterium & Rhodocyclales & 0.195 & 0.189 \\
\hline 19. & $\begin{array}{l}\text { Acetobacter, Acetobacteraceae, Gluconacetobacter, Inquilinus, Rhodospirillaceae }(1.291 \%, 0.028 \%) \text {, } \\
\text { Rhodospirillales, Roseococcus, Roseomonas, Skermanella }\end{array}$ & Rhodospirillales & 1.485 & 1.286 \\
\hline 20. & Mitochondria, Rickettsiales & Rickettsiales & 0.024 & 0.154 \\
\hline 21. & $\begin{array}{l}\text { Blastomonas, Erythrobacteraceae, Novosphingobium, Kaistobacter, Sphingobium }(0.102 \%, 1.554 \%), \\
\text { Sphingomonadaceae, Sphingomonas, Sphingomonas echinoides, Sphingomonas wittichii (0.201\%,1.666\%), } \\
\text { Sphingopyxis alaskensis }\end{array}$ & Sphingomonadales & 3.538 & 5.646 \\
\hline 22. & Spirobacillales & Spirobacillales & 0.039 & 0.014 \\
\hline 23. & Syntrophobacteraceae $(1.555 \%, 0.005 \%)$ & Syntrophobacterales & 1.555 & 0.005 \\
\hline 24. & Piscirickettsiaceae & Thiotrichales & 0.289 & \\
\hline 25. & Arenimona, Dokdonella, Luteibacter rhizovicinus, Lysobacter, Lysobacter brunescens, Nevskiaramosa, & Xanthomonadales & 3.241 & 11.194 \\
\hline
\end{tabular}

Members of Chryseobacterium have so far been found in various environments, i.e., freshwater, soil and sludge, rhizosphere and phyllosphere, midgut of insects, faeces of millipede, raw fish, chicken and dairy products, or clinical samples. On poplar, Chryseobacterium has been detected on two hybrid clones, where it was classified as an endophyte (Ulrich et al. 2008).
Flavobacteria are generally known as common, free living organisms in soil and water. Most species are harmless, psychrotolerant, present in temperate to polar regions, and particularly in low salinity ecosystems (Kolton et al. 2016). Several species are infectious to freshwater fish and humans (Berg et al. 2005). Little is known about the occurrence of Flavobacterium in plants. The first evidence of 
Table 1 (continued)

\begin{tabular}{|c|c|c|c|c|}
\hline & $\begin{array}{l}\text { Pseudoxanthomonas, Rhodanobacter, Sinobacteraceae }(1.487 \%, 0.035 \%) \text {, Stenotrophomonas, } \\
\text { Stenotrophomonas acidaminiphila, Thermomonas, Steroidobacter, Xanthomonadaceae }(1.401 \%, 8.410 \%) \text {, } \\
\text { Xanthomonas }\end{array}$ & & & \\
\hline & Frequency & & 36.872 & 73.785 \\
\hline & Number of taxa (143) & & 129 & 109 \\
\hline \multicolumn{5}{|c|}{ Phylum Spirochaetes } \\
\hline \multirow[t]{3}{*}{1.} & Turneriella & Leptospirales & 0.010 & 0.010 \\
\hline & Frequency & & 0.010 & 0.010 \\
\hline & Number of taxa (1) & & 1 & 1 \\
\hline \multicolumn{5}{|c|}{ Phylum Verrucomicrobia } \\
\hline 1. & Chthoniobacteraceae $(1.587 \%, 0.011 \%)$ & Chthoniobacterales & 1.766 & 0.018 \\
\hline 2. & Luteolibacter, Methylacidiphilae & Verrucomicrobiales & 0.490 & 0.505 \\
\hline 3. & Opitutaceae & Opitutales & 0.640 & 0.003 \\
\hline 4. & Pedosphaera $(2.065 \%, 0.002 \%)$ & Pedosphaerales & 2.166 & 0.002 \\
\hline \multirow[t]{15}{*}{5.} & Prosthecobacter debontii, Verrucomicrobia & Verrucomicrobiales & 0.245 & 0.301 \\
\hline & Frequency & & 5.307 & 0.829 \\
\hline & Number of taxa (12) & & 12 & \\
\hline & Non-identified bacteria - frequency & & 3.431 & 0.164 \\
\hline & Number of taxa (102) & & 82 & 16 \\
\hline & Bacteria absent in NCBI database- frequency & & 0.701 & 0.630 \\
\hline & Number of taxa (243) & & 242 & \\
\hline & & & \multicolumn{2}{|c|}{ Number } \\
\hline & OTU & & $34866^{b}$ & $21207^{\mathrm{b}}$ \\
\hline & Taxa & & $615^{\mathrm{b}}$ & $249^{\mathrm{b}}$ \\
\hline & Margalef's diversity index $-D_{M q}$ & & 58.7039 & 24.8944 \\
\hline & Shannon's diversity index $-H^{\prime}$ & & 4.1276 & 3.1787 \\
\hline & Simpson's diversity index $-D$ & & 0.6428 & 0.5761 \\
\hline & Shannon's evenness index $-E$ & & 0.0294 & 0.0716 \\
\hline & Berger-Parker's dominance index $-d$ & & 0.0888 & 0.1530 \\
\hline
\end{tabular}

\begin{tabular}{llll}
\hline 0 & 14 & 38 & - diversity of bacterial community (\%)
\end{tabular}

Explanations

${ }^{a}$ Indicates frequency in soil and wood, respectively

${ }^{\mathrm{b}}$ Indicates a statistically significant difference according to $\chi^{2}$ test, $P<0.001$

Flavobacterium in plants was from a study of the barley rhizosphere (Johansen et al. 2002). Recently, Kolton et al. (2016) detected epiphytic and endophytic Flavobacteria on/ in roots and leaves of cucumber, lettuce, maize, peanuts, peppers, tomatoes, thale cress [Arabidopsis thaliana (L.) Heynh.] and wheat. They may be associated with plant health. They move rapidly over solid surfaces due to unique gliding-motility (linked to a novel type IX secretion system), enabling fast propagation and colonization (McBride and Zhu 2013; McBride and Nakane 2015). Flavobacterium johnsoniae causes a 'soft-rot' of various fresh plants (Liao and Wells 1986). Flavobacterium abundance can vary considerably and is a function of plant and environment interactions. So far there was no information on occurrence of Flavobacterium in forest habitats. Our record is the first. Its occurrence could possibly have resulted from the slightly less acid soil ( $\mathrm{pH}$ 6.5) of the diseased poplar plantation.

Enterobacteriales are facultative anaerobes, common in water or soil, or are parasites of animals and plants. They can live on/in wood of poplar, and even much longer than on wood of other tree species (Schönwälder et al. 2002; Milling et al. 2005). The most abundant representatives of Enterobacteriales were Serratia and Sodalis (15.3\% and
$6.5 \%$ in wood). Serratia is often a harmless, environmental, plant-associated endophyte or free-living bacterium, found in decaying plants or animals and humans. Many Serratia species have plant growth-promoting (PGP) ability and have been developed as biocontrol agents for soil-borne fungal pathogens (Hallmann et al. 1997).

The very fast development of wilt in the poplar trees studied suggests that pathogenic Pseudomonas, i.e., P. syringae and $P$. aeruginosa, may be involved in development of disease. Pseudomonas occurred relatively abundantly in soil from where they could infect roots. The short span of the disease in the surveyed poplars may also have resulted from a quick response of the host plant. It is known that perception of Pseudomonas by plants occurs quickly. Changes in plant signal transduction and in plant gene expression occur within 2 and $15 \mathrm{~min}$, respectively, after infection and exposure to bacterial elicitors (Gómez-Gómez et al. 1999; de Torres et al. 2003). The first cellular symptoms are observed within $5 \mathrm{~h}$ (Bestwick et al. 1997). Bacteria live on nutrients present in the apoplast of host cells, the acidic components of cell walls and nutrients in dying cells (Preston 2004). Apart from changes in the plant's physiology, Pseudomonas can cause mechanical damage of the host tissues through 
the ice nucleation (Wisniewski et al. 1997). They may contribute to the final disease effect. The second potentially pathogenic species, $P$. aeruginosa, is an environmental organism that can survive in different conditions and is particularly well-adapted to wet and damp habitats, i.e., soil, aqueous solutions (Jefferies et al. 2012), and thus probably also in sap oozing from wounds on the diseased poplars. It forms biofilms which increase its persistence and stability and generate extensive genetic diversity which enables the bacteria to persist and spread under different environmental stresses (Webb et al. 2003). The genus Pseudomonas may also include neutral or beneficial strains, i.e., with biocontrol- or plant growth-promoting activity, or capable of inducing systemic plant defense. One of those is Pseudomonas fluorescens Migula, usually very common in soil and rhizosphere, and on plant surfaces (Silby et al. 2009). Degradative enzymes determine the virulence of pathogenic strains (Preston 2000). However, the same enzymes are produced by plant growth-promoting Pseudomonas. Thus, distinguishing between pathogenic and beneficial interactions needs recognition of: (1) the specificity and combination of extracellular compounds produced by the bacterium, (2) effects of habitat conditions (temperature, moisture) on host and bacterium, (3) host genotype, (4) host physiology, (5) the ability of the bacterium to respond to host recognition (Preston 2004). Success for the plant depends on its ability to distinguish beneficial symbionts and harmless saprotrophs from pathogenic parasites, and in using induced defense responses to eliminate dangerous pathogens at minimum cost.

Rhizobiales are well-known, beneficial, microsymbiotic, legume-nodulating, nitrogen-fixing, methanotrophic bacteria providing nutrients, vitamins, phytohormones (auxins and cytokinins) and precursors of essential plant metabolites (Vorholt 2012).

Nitrogen fixation by Rhizobiales has recently been found also in non-leguminous plants (Fischer et al. 2012), including wild poplar harbouring diazotrophic bacteria. However, poplar can be a natural host of pathogenic $R$. radiobacter, the occurrence of which in the microbiome of the studied poplars cannot be excluded, although the typical symptoms of $R$. radiobacter, i.e., tumour-like growths (galls) on stems and roots, often above ground, were not observed.

Sphingobacteriales are common in soil and marine habitats. One member of this order, Flavitalea populi, has been isolated from soil of a Euphrates poplar (Populus euphratica Oliv.) forest (Wang et al. 2011).

Xanthomonadales are typically rod-shaped, obligate aerobes with optimal growth at $25-30{ }^{\circ} \mathrm{C}$. There are at least 27 plant-associated Xanthomonas species, which colonize at least 400 plant species (An et al. 2019). Xanthomonas populi can cause wilting, necrosis, rot and injury of poplar. It initially feeds on living host tissue and kills the tree in later stages of infection. Xanthan, a biopolymer, contributes to formation of biofilm which masks the bacteria, preventing recognition and early response from plant defense mechanisms (Büttner and Bonas 2010).

Betaproteobacteria, common in the soil samples, occupy diverse habitats and have various metabolic strategies. They may be autotrophic, heterotrophic and diazotrophic. Some, including Bordetella, Burkholderia and Ralstonia, are pathogenic on plants (Dworkin et al. 2006). However, the volatile organic compounds of Burkholderia pyrrocinia strain JK-SH007 inhibit three poplar canker pathogens: Cytospora chrysosperma (Pers.) Fr., Phomopsis macrospora Tak. Kobay. and Chiba, and Fusicoccum aesculi Corda) (Liu et al. 2020).

Dubious and unexpected species include Pedobacter cryoconitis ( $7.306 \%$ in wood) and Sodalis (6.524\% in wood). Pedobacter cryoconitis is a rod-shaped bacterium, facultatively aerobic and psychrophilic. Other Pedobacter species have been isolated from soil and compost, water and freshwater-lake sediment, and plant rhizosphere (Kwon et al. 2007, 2011). So far, Pedobacter was not found in the forest habitat. However, the bacterium is a cellulose decomposer, and this explains its abundant occurrence in wood. Endosymbiontic Sodalis has so far been associated with spittlebugs and feather-chewing bird lice, and a human wound.

Many bacteria detected were possibly the initial endophytes in a variety of tissues in trees that were, so far, healthy. They probably do not participate in the initial development of disease but become pathogenic later, after development of disease started by fungi (Kwaśna et al. 2021). They need favorable conditions, including lower temperature and higher humidity of soil from where they spread to plants. Their adaptation strategies for growth, production and activation of enzymes could compensate for the occasionally negative effects of the habitat.

Plants can be colonized simultaneously by a large variety of bacteria (Bacon and Hinton 2006). Most studies on bacterial endophytes were done on agricultural and horticultural plants. However, endophytic bacteria have also been detected in trees: elm, pine, oak, citrus and coffee (Bacon and Mead 1971; Mocali et al. 2003; Vega et al. 2005). Their survival was strongly dependent on moisture.

Endophytic bacteria in poplar have rarely been studied. Single studies have described only specific strains (Germaine et al. 2004; Van Aken et al. 2004; Doty et al. 2005). More recently, Moore et al. (2006) and Ulrich et al. (2008) studied the diversity of endophytic bacteria in Рориlus growing in contaminated and non-contaminated fields. They reported a high diversity and domination of Gammaproteobacteria (28-61\%), especially Pseudomonas spp. (19-46\%). In the present study Pseudomonas frequency was 3-9\%, and other Gammaproteobacteria were sporadic in soil $(0.01 \%)$ and absent in wood. The differences in abundance and diversity may result from the genetic background of the 
host tree (Ulrich et al. 2008). Each tree species or clone has an association with specific bacterial endophytes (Cambours et al. 2005; Moore et al. 2006) and the dependence of endophytic bacterial communities on host genotype seems to be stronger in longer-lived trees. In the endophytic phase, bacteria may have beneficial effects on their hosts and may play an important role in plant physiology, including resistance. Apart from Pseudomonas, other bacteria detected in soil or wood of the poplars studied, i.e., Actinobacteria, Bacillus, Burkholderia and Chryseobacterium, promote plant growth by elimination of pathogenic microorganisms, synthesis of growth-stimulating plant hormones, low-molecular compounds or enzymes, and creation of plant disease resistance, particularly during periods of drought or nutrient deprivation (Shin et al. 2007; Montero-Calasanz et al. 2013; Timm et al. 2016). Their presence in soil is explained by their ability to live on organic debris as saprotrophs. They usually colonize the host plant from the rhizosphere soil. Their adaptation to an endophytic or pathogenic lifestyle in plants results from their ability to pass (actively or passively) through the endodermis and pericycle and enter the xylem vessels (Compant et al. 2010).

\section{Conclusions}

New diseases, including wilt, necrosis of stems and dieback may appear in forests and plantations of trees. Рориlus hybrids may be subjected to various, so far unidentified pathogenic agents, including bacteria. Bacteria can contribute to the development of disease, but can also have an important role in limiting or preventing the development of initial pathogens. This situation can lead to near-total disappearance of some diseases and sudden emergence of new pathogens. Poplar wilt symptoms may be a consequence of various factors, the most important being climate and its effects on development of pathogens and the host-pathogen relationship. New diseases can spread from soil or from introduced plant material, the latter potentially introducing them into new areas.

Author contributions Conceptualization WS and JB-B; methodology JB-B, formal analysis MB and JB-B, investigation HK and JB-B; resources WS and JB-B, writing, original draft preparation $\mathrm{HK}$, writing, review and editing HK, visualization JB-B, supervision HK, project administration WS. All authors have read and agreed to the published version of the manuscript.

Funding Not applicable.

Availability of data and materials Data associated with a paper is available at https://figshare.com/s/dd06afb0fb7d348f5388?fbclid= IwAR15_FXPrsPve6TSo4cBUFtNLR655ZE6FFjpK1X80TVr706IDDTb6dVcco.
Code availability Not applicable.

\section{Declarations}

Conflict of interest The authors declare no conflict of interest. The funders had no role in the design of the study, in the collection, analyses, or interpretation of data, in the writing of the manuscript, or in the decision to publish the results.

Ethics approval Not applicable.

Consent to participate Not applicable.

Consent for publication Not applicable.

Open Access This article is licensed under a Creative Commons Attribution 4.0 International License, which permits use, sharing, adaptation, distribution and reproduction in any medium or format, as long as you give appropriate credit to the original author(s) and the source, provide a link to the Creative Commons licence, and indicate if changes were made. The images or other third party material in this article are included in the article's Creative Commons licence, unless indicated otherwise in a credit line to the material. If material is not included in the article's Creative Commons licence and your intended use is not permitted by statutory regulation or exceeds the permitted use, you will need to obtain permission directly from the copyright holder. To view a copy of this licence, visit http://creativecommons.org/licenses/by/4.0/.

\section{References}

An SQ, Potnis N, Dow M, Vorhölter FJ, He YQ, Becker A, Teper D, Li Y, Wang N, Bleris L, Tang J-L (2019) Mechanistic insights into host adaptation, virulence and epidemiology of the phytopathogen Xanthomonas. FEMS Microbiol Rev 44(1):1-32

Attila C, Ueda A, Cirillo SL, Cirillo JD, Chen W, Wood TK (2008) Pseudomonas aeruginosa PAO1 virulence factors and poplar tree response in the rhizosphere. Microb Biotechnol 1(1):17-29. https://doi.org/10.1111/j.1751-7915.00002.x

Bacon CW, Hinton DM (2006) Bacterial Endophytes: the endophytic niche, its occupants and its utility. In: Gnanamanickam SS (ed) Plant-associated bacteria. Springer, The Netherlands, pp 155-194

Bacon M, Mead CE (1971) Bacteria in the wood of living aspen, pine and alder. Northwest Sci 45:270-275

Bengtsson-Palme J, Veldre V, Ryberg M, Hartmann M, Branco S, Wang Z, Nilsson RH (2013) Improved software detection and extraction of ITS1 and ITS2 from ribosomal ITS sequences of fungi and other eukaryotes for analysis of environmental sequencing data. Methods Ecol Evol 4:914-919. https://doi.org/10.1111/ 2041-210X.12073

Berg G, Eberl L, Hartmann A (2005) The rhizosphere as a reservoir for opportunistic human pathogenic bacteria. Environ Microbiol 7:1673-1685

Bestwick CS, Brown IR, Bennett MHR, Mansfield JW (1997) Localisation of hydrogen peroxide accumulation during the hypersensitive reaction of lettuce cells to Pseudomonas syringae pv. phaseolicola. Plant Cell 9:209-221

Biosca E, Martín S, Zuriaga P, Monton C, Lopez-Ocana L, Lopez M (2006) Characterization of Brenneria sp. from poplar cankers in Spain. In: Mendez-Vilas A (ed) Modern multidisciplinary applied microbiology, pp 385-389 
Büttner D, Bonas U (2010) Regulation and secretion of Xanthomonas virulence factors. FEMS Microbiol Rev 34(2):107-133. https:// doi.org/10.1111/j.1574-6976.2009.00192.x

Cambours MA, Nejad P, Granhall U, Ramstedt M (2005) Frost-related dieback of willows. Comparison of epiphytically and endophytically isolated bacteria from different Salix clones, with emphasis on ice nucleation activity, pathogenic properties and seasonal variation. Biomass Bioenergy 28:15-27

Cole JR, Wang Q, Fish JA, Chai B, Mc Garrell DM, Sun Y, Tiedje JM (2014) Ribosomal database project: data and tools for high throughput rRNA analysis. Nucleic Acids Res 42:D633-D642

Compant S, Clément C, Sessitsch A (2010) Plant growth-promoting bacteria in the rhizo- and endosphere of plants: their role, colonization, mechanisms involved and prospects for utilization. Soil Biol Biochem 42:669-678

de Torres M, Sanchez P, Fernandez-Delmond I, Grant M (2003) Expression profiling of the host response to bacterial infection: the transition from basal to induced defence responses in RPM1-mediated resistance. Plant J 33:665-676

Doty SL, Dosher MR, Singleton GL, Moore AL, Van Aken B, Stettler RF, Strand SE, Gordon MP (2005) Identification of an endophytic Rhizobium in stems of Populus. Symbiosis 39:27-35

Dworkin M, Falkow S, Rosenberg E, Schleifer KH, Stackebrandt E (2006) The Prokaryotes, volume 5-proteobacteria: alpha and beta subclasses, vol 11, 3rd ed. Springer, Berlin. https://doi.org/ 10.1007/0-387-30745-1

Fischer D, Pfitzner B, Schmid M, Simões-Araújo JL, Reis VM, Pereira W, Ormeño-Orrillo E, Hai B, Hofmann A, Schloter M, Martinez-Romero E, Baldani JI, Hartmann A (2012) Molecular characterisation of the diazotrophic bacterial community in uninoculated and inoculated field-grown sugarcane (Saccharum sp.). Plant Soil 356:83-99. https://doi.org/10.1007/ s11104-011-0812-0

Germaine K, Keogh E, Garcia-Cabellos G, Borremans B, Lelie D, Barac T, Oeyen L, Vangronsveld J, Moore FP, Moore ER, Campbell CD, Ryan D, Dowling DN (2004) Colonisation of poplar trees by gfp expressing bacterial endophytes. FEMS Microbiol Ecol. 48(1):109-18. https://doi.org/10.1016/j.femsec. 2003.12.009 PMID: 19712436. Accessed 1 Apr 2004

Gómez-Gómez L, Felix G, Boller T (1999) A single locus determines sensitivity to bacterial flagellin in Arabidopsis thaliana. Plant J 18:277-284

Gweon HS, Oliver A, Taylor J, Booth T, Gibbs M, Read DS, Schonrogge K (2015) PIPITS: An automated pipeline for analyses of fungal internal transcribed spacer sequences from the Illumina sequencing platform. Methods Ecol Evol 6(8):973-980. https:// doi.org/10.1111/2041-210X.12399

Hallmann J, Quadt-Hallmann A, Mahaffee WF, Kloepper JW (1997) Bacterial endophytes in agricultural crops. Can J Microbiol 43:895-914

Jansson S, Douglas CJ (2007) Populus: a model system for plant biology. Annu Rev Plant Biol 58:435-458. https://doi.org/10. 1146/annurev.arplant.58.032806.103956

Jefferies JMC, Cooper T, Yam T, Clarke SC (2012) Pseudomonas aeruginosa outbreaks in the neonatal intensive care unit-a systematic review of risk factors and environmental sources. J Med Microbiol 61:1052-1061

Johansen JE, Binnerup SJ, Lejbølle KB, Mascher F, Sørensen J, Keel C (2002) Impact of biocontrol strain Pseudomonas fluorescens CHA0 on rhizosphere bacteria isolated from barley (Hordeum vulgare L.) with special reference to Cytophaga-like bacteria. J Appl Microbiol 93(6):1065-1074

Kolton M, Erlacher A, Berg G, Cytryn E (2016) The Flavobacterium genus in the plant holobiont: ecological, physiological, and applicative insights. Springer Science + Business Media Singapore, Singapore
Kwaśna H, Szewczyk W, Baranowska M, Gallas E, Wiśniewska M, Behnke-Borowczyk B (2021) Mycobiota associated with vascular wilt of poplar. Plants 10(5):892. https://doi.org/10.3390/ plants 10050892

Kwon SW, Kim BY, Lee KH, Jang KY, Seok SJ, Kwon JS, Kim WG, Weon HY (2007) Pedobacter suwonensis sp. nov., isolated from the rhizosphere of Chinese cabbage (Brassica campestris). Int J Syst Evol Microbiol 57:480-484

Kwon SW, Son JA, Kim SJ, Kim YS, Park IC, Bok JI, Weon HY (2011) Pedobacter rhizosphaerae sp. nov. and Pedobacter soli sp. nov., isolated from the rhizosphere soil of Chinese cabbage (Brassica campestris). Int J Syst Evol Microbiol 61:2874-2879

Li A, He W (2019) Molecular aspects of an emerging poplar canker caused by Lonsdalea populi. Front Microbiol 10:2496

Li Y, He W, Ren F, Guo L, Chang J, Cleenwerck I, Ma Y, Wang H (2014) A canker disease of Populus $\times$ euramericana in China caused by Lonsdalea quercina subsp. populi. Plant Dis 98:368378. https://doi.org/10.1094/PDIS-01-13-0115-RE

Liao C-H, Wells JM (1986) Properties of Cytophaga johnsonae strains causing spoilage of fresh produce at food markets. Appl Environ Microbiol 52:1261-1265

Liu A, Zhang P, Bai B, Bai F, Jon T, Ren J (2020) Volatile organic compounds of endophytic Burkholderia pyrrocinia strain JK-SH007 promote disease resistance in poplar. Plant Dis 104(6):1610-1620

Magurran AE (1988) Ecological diversity and its measurement. Princeton University Press, Princeton, pp 1-180

McBride MJ, Nakane D (2015) Flavobacterium gliding motility and the type IX secretion system. Curr Opin Microbiol 28:72-77

McBride MJ, Zhu YT (2013) Gliding motility and Por secretion system genes are widespread among members of the phylum Bacteroidetes. J Bacteriol 195:270-278

Milling A, Kehr R, Wulf A, Smalla K (2005) Survival of bacteria on wood and plastic particles: dependence on wood species and environmental conditions. Holzforschung 59(1):72-81

Mocali S, Bertelli E, Di Cello F, Mengoni A, Sfalanga A, Viliani F, Caciotti A, Tegli S, Surico G, Fani R (2003) Fluctuation of bacteria isolated from elm tissue during different seasons and from different plant organs. Res Microbiol 154:105-114

Montero-Calasanz MC, Göker M, Rohde M, Spröer C, Schumann P, Busse H-J, Schmid M, Tindall B, Klenk H-P, Camacho M (2013) Chryseobacterium hispalense sp. nov., a plant-growth-promoting bacterium isolated from a rainwater pond in an olive plant nursery, and emended descriptions of Chryseobacterium defluvii, Chryseobacterium indologenes, Chryseobacterium wanjuense and Chryseobacterium gregarium. Int J Syst Evol Microbiol. https:// doi.org/10.1099/ijs.0.052456-0

Moore PF, Barac T, Borremans B, Oeyen L, Vangronsveld J, Van der Lelie D, Campbell CD, Moore ERB (2006) Endophytic bacterial diversity in poplar trees growing on a BTEX-contaminated site: the characterization of isolates with potential to enhance phytoremediation. Syst Appl Microbiol 29:539-556

Nesme X, Steenackers M, Steenackers V, Picard C, Ménard M, Ridé S, Ridé M (1994) Differential host-pathogen interactions among clones of poplar and strains of Xanthomonas populi pv. populi. Phytopathology 84:101-107

Newcombe G (1996) The specificity of fungal pathogens of Populus. In: Stettler RF, Bradshaw HD, Heilman PE, Hinckley TM (eds) Biology of Populus and its implications for management and conservation. NRC Research Press, Ottawa, pp 223-246

Preston GM (2000) Pseudomonas syringae pv. tomato: the right pathogen of the right plant at the right time. Mol Pl Pathol 1:263-275

Preston GM (2004) Plant perceptions of plant growth-promoting Pseudomonas. Phil Trans R Soc Lond B 359:907-918

Ramstedt M, Aström B, Von Fircks HA (1994) Dieback of poplar and willow caused by Pseudomonas syringae in combination with freezing stress. Eur J Forest Pathol 24:305-315 
Ridé M (1993) Xanthomonas populi: cause of bacterial canker of poplar. In: Swing JG, Civerol EL (eds) Xanthomonas. Chapman and Hall, London, pp 64-69

Schönwälder A, Kehr R, Wulf A, Smalla K (2002) Wooden boards affecting the survival of bacteria? Holz Als Roh-Und Werkstoff 60:249-257

Schulz B, Römmert A-K, Dammann U, Aust H-J, Strack D (1999) The endophyte-host interaction: a balanced antagonism? Mycol Res 103(10):1275-1283

Shin DS, Park MS, Jung S, Lee MS, Lee KH, Bae KS, Kim SB (2007) Plant growth-promoting potential of endophytic bacteria isolated from roots of coastal sand dune plants. J Microbiol Biotechnol 17:1361-1368

Silby MW, Cerdeno-Tarraga AM, Vernikos GS, Giddens SR, Jackson RW, Preston GM, Zhang XX, Moon CD, Gehrig SM, Godfrey SA, Knight CG, Malone JG, Robinson Z, Spiers AJ, Harris S, Challis GL, Yaxley AM, Harris D, Seeger K, Murphy L, Rutter S, Squares R, Quail MA, Saunders E, Mavromatis K, Brettin TS, Bentley SD, Hothersall J, Stephens E, Thomas CM, Parkhill J, Levy SB, Rainey PB, Thomson NR (2009) Genomic and genetic analyses of diversity and plant interactions of Pseudomonas fluorescens. Genome Biol 10:R51. https://doi.org/10.1186/gb-2009-10-5-r51

Timm CM, Pelletier DA, Jawdy SS, Gunter LE, Henning JA, Engle N, Aufrecht J, Gee E, Nookaew I, Yang Z, Lu TY, Tschaplinski TJ, Doktycz MJ, Tuskan GA, Weston DJ (2016) Two poplarassociated bacterial isolates induce additive favorable responses in a constructed plant-microbiome system. Front Plant Sci 7:497. https://doi.org/10.3389/fpls.00497 (PMID:27200001;PMCID: PMC4845692)

Toth T, Lakatos T, Koltay A (2013) Lonsdalea quercina subsp. populi subsp. nov., isolated from bark canker of poplar trees. Int J Syst Evol Microbiol 63:2309-2313. https://doi.org/10.1099/ijs.0. 042911-0

Ulrich K, Ulrich A, Ewald D (2008) Diversity of endophytic bacterial communities in poplar grown under field conditions. FEMS Microbiol Ecol 63:169-180
Van Aken B, Peres CM, Doty SL, Yoon JM, Schnoor JL (2004) Methylobacterium populi sp. nov., a novel aerobic, pink-pigmented, facultatively methylotrophic, methane-utilizing bacterium isolated from poplar trees (Populus deltoides $\times$ nigra DN34). Int J Syst Evol Microbiol 54:1191-1196

Vega FE, Pava-Ripoll M, Posada F, Buyer YS (2005) Endophytic bacteria in Coffea arabica L. J Basic Microbiol 45:371-380

Vorholt JA (2012) Microbial life in the phyllopshere. Nat Rev Microbiol 10:828-840

Wang Q, Garrity GM, Tiedje JM, Cole JR (2007) Naive Bayesian Classifier for rapid assignment of rRNA sequences into the new bacterial taxonomy. Appl Environ Microbiol 73(16):5261-5267

Wang Y, Cai F, Tang Y, Dai J, Qi H, Rahman E, Peng F, Fang C (2011) Flavitalea populi gen. nov., sp. nov., isolated from soil of a Euphrates poplar (Populus euphratica) forest. Int J Syst Evol Microbiol 61:1554-1560

Webb JS, Thompson LS, James S, Charlton T, Tolker-Nielsen T, Koch B, Givskov M, Kjelleberg S (2003) Cell death in Pseudomonas aeruginosa biofilm development. J Bacteriol 185:4585-4592

Wisniewski M, Lindow SE, Ashworth EN (1997) Observations of ice nucleation and propagation in plants using infrared video thermography. Pl Physiol 113:327-334

Yuki IY, Fumiko T, Takafumi M (2013) Pathogenicity and virulence factors of Pseudomonas syringae. J Gen Plant Pathol 79(5):285-296

Zhang J, Kobert K, Flouri T, Stamatakis A (2014) PEAR: a fast and accurate illumina paired-end reAd mergeR. Bioinformatics 30(5):614-620. https://doi.org/10.1093/bioinformatics/btt593

Publisher's Note Springer Nature remains neutral with regard to jurisdictional claims in published maps and institutional affiliations. 